\title{
GIẢNG DẠY VỀ TẠO ĐộNG LỰC NHÓM : KINH NGHIỆM TRONG VIỆC XÂY DỬNG CHƯƠNG TRİNH NÀY CHO SINH VIÊN CHUYÊN NGÀNH CÔNG NGHỆ CHẾ TẠO MÁY
}

\author{
Phan Hoàng Danh ${ }^{(*)}$
}

(*) Thạc sĩ. Truò̀ng Đại học Công nghệ Đồng Nai. Email: phanhoangdanh@dntu.edu.vn

DOI: $10.37550 /$ tdmu.CFR/2021.01.117

\section{Tóm tắt}

Sự phát triển của các hoạt động tạo động lực nhóm cho sinh viên chuyên ngành công nghệ chế tạo máy tại truòng Đại học Công nghệ Đồng Nai được trình bày dựa trên sụ phản hồi của sinh viên và các bài học trọng tâm trong họ phần/môn học này. Thay vì để sinh viên tự học các kỹ năng làm việc nhóm, việc giảng dạy chính thức về kỹ năng này " tạo động lực nhóm " được đưa vào giảng dạy trục tiếp nhu một môn học chính trong chuong trình dành cho sinh viên năm hai chuyên ngành công nghệ chế tạo máy tại DNTU nhiều năm qua. Trong thời gian đầu áp dụng chưong trình này, sinh viên tỏ ra không quan tâm và phản đối với việc học môn học này rộng rãi. Tuy nhiên, nội dung và cách truyền tải tài liệu môn học đã tạo ra một bước ngoặc đáng mong đợi, gần $80 \%$ sinh viên cho rằng môn hoc này rất có giá trị và rất hưu ích với họ. Các yếu tố tạo nên thành công này liên quan đến việc trình bày tài liệu một cách trục quan và tức thì đến tùng sinh viên và các dụ án ho đang làm cũng nhu cung cấp tài liệu xuyên suốt cho họ trong suốt quá trình học.

Từ khóa: Động lục nhóm, giáo dục kỹ thuật, nhóm kỹ thuật.

\section{1. Đặt vấn đề}

Làm việc nhóm là điều cần thiết trong quá trình sinh viên theo học tại môi trường đại học, đặc biệt là các bạn sinh viên chuyên ngành kỹ thuật, việc học nhóm sẽ giúp sinh viên hỗ trợ cho nhau trong việc học, tiết kiệm thời gian mà hiệu quả lại cao [1] [2]. Kỹ năng làm việc nhóm là một trong những yêu cầu để sinh viên tốt nghiệp kỹ sư tại trường Đại học Công Nghệ Đồng Nai phải đạt được trước khi hoàn thành chương trình học tại trường để tốt nghiệp. Với việc đưa vào giảng dạy chính thức kỹ năng làm việc nhóm, sinh viên tốt nghiệp có thể hoạt động hiệu quả như một cá nhân hoặc người lãnh đạo trong các nhóm một cách đa dạng [3] [4]. Thông thường, trong các môn học về chuyên ngành kỹ thuật, sinh viên làm việc nhưng sẽ không được hướng dẫn cụ thể về cách hoạt động của một nhóm thay vào đó, các nhóm sẽ tự tìm hiểu về động lực của nhóm và vai trò của người hướng dẫn với các vấn 
đề khi chúng phát sinh. Việc dạy tạo động lực nhóm cho sinh viên khối ngành xã hội thường dễ dàng hơn so với các bạn sinh viên học chuyên ngành kỹ thuật [5].

Tuy nhiên, khi đưa vào giảng dạy trực tiếp như một môn học chính trong chương trình dành cho sinh viên năm hai chuyên ngành công nghệ chế tạo máy tại DNTU, đã thu hút sự chú ý và quan tâm đối với khóa học này từ các bạn sinh viên chuyên ngành kỹ thuật, các bạn sinh viên đã bị ảnh hưởng tích cực sau một tuần khi được học về các kỹ năng làm việc nhóm, sự cần thiết của làm việc nhóm khi các bạn ra trường, về sự phát triển nghề nghiệp, định hướng nghề nghiệp trong tương lai. Hơn nữa, qua quá trình học những sinh viên ban đầu có xu hướng phản đối đưa môn học này vào chương trình học lại là những người tham gia học tích cực nhất. Những giảng viên tham gia giảng dạy cũng báo cáo các kết quả đạt được là rất tốt. Sự thành công trong việc hướng dẫn cho sinh viên về kỹ năng tạo động lực nhóm đã mang đến cho chương trình đào tạo khối ngành kỹ thuật được cải tiến một cách hiệu quả, môn học này chính thức như một phần của chương trình giảng dạy tại trường [6] [7] [8]. Cơ bản nhất trong việc dạy động lực học nhóm là làm thế nào để cân bằng và tích hợp giữa học tập kiến thức chuyên môn về kỹ thuật và kỹ năng mềm trong giao tiếp vì theo kinh nghiệm qua các khóa đào tạo trước đây tại trường, khi giảng dạy về tạo động lực nhóm chưa được áp dụng vào chương trình học, phần lớn sinh viên kỹ thuật hầu như hoàn toàn bác bỏ hoặc phản đối các hoạt động học tập đi sâu vào lĩnh vực xã hội, giao tiếp hoặc các môn học không phải kỹ thuật.

Theo kết quả khảo sát thực hiện tại trường DNTU, sinh viên cần có kỹ năng làm việc nhóm, kết quả sau khi đưa vào giảng dạy cho thấy có thể dạy và phát triển kỹ năng này như một môn học chính thức trong chương trình đào tạo. Thách thức đối với giảng viên là làm sao trình bày bài giảng, tài liệu học đến sinh viên một cách dễ dàng và dễ hiểu, sinh viên khối ngành kỹ thuật sẽ đánh giá cao và đón nhận. Trong thời gian các học kỳ năm học 2018 sinh viên không quan tâm đến nội dung và chương trình học này. Bắt đầu từ 2019 những thay đổi đáng kể đối với chương trình giảng dạy đã được thông qua bao gồm việc tăng cường nhấn mạnh vào làm việc nhóm, hiệu quả của kỹ năng này khi phỏng vấn xin việc, sự nhấn mạnh vào tính năng động của nhóm và nội dung bài học đã làm cho đại đa số sinh viên hiện nay thấy rằng việc học này là rất có giá trị và hữu ích.

\section{Bối cảnh}

\subsection{Chương trình giảng dạy cho sinh viên chuyên ngành chế tạo máy}

Trước năm 2018, chương trình giảng dạy chuyên ngành chế tạo máy năm thứ hai tại Đại học Công nghệ Đồng Nai (DNTU) được quy ước theo khung chương trình giảng dạy, một năm bao gồm 3 học kỳ, mỗi học kỳ đăng ký tối thiểu là 9 tín chỉ và tối đa là 16 tín và xen kẽ các môn học lý thuyết là làm đồ án thực tế. Các đồ án môn học được thực hiện thiết kế, xây dựng và vận hành sau đó được thuyết trình và báo cáo bằng văn bản. Bắt đầu từ năm 2018, những thay đổi đáng kể đối với toàn bộ chương trình giảng dạy đã được thông qua với sự ra đời của một chương trình tích hợp [10] [11]. Chương trình chú trọng nhiều hơn vào thiết kế, sinh viên học về lý thuyết và ngay lập tức áp dụng nó, cùng với việc vận dụng kiến thức từ các khóa học đầu tiên của họ để đưa vào trong đồ án. Các đồ án sẽ được cạnh tranh giữa tất cả các đội sau đó là cuộc thi nói sẽ được thuyết trình và nộp các báo cáo 
chính thức bằng văn bản. Sau 10 tuần hướng dẫn trong một khóa học, $50 \%$ của mỗi sinh viên có điểm dựa trên các yếu tố cá nhân (chuyên cần, kiểm tra giữa kỳ, kiểm tra cuối kỳ) và $50 \%$ dựa trên các yếu tố nhóm ( 1 đồ án và 10 bài tập hướng dẫn). Điểm của nhóm được điều chỉnh bởi điểm đánh giá cá nhân từng sinh viên.

\subsection{Thành lập nhóm}

Khóa học cơ khí chế tạo có khoảng 120 học viên được chia thành 20 nhóm 5 hoặc 6 sinh viên, các nhóm được thành lập bởi người hướng dẫn [12] để tối đa hóa sự đa dạng [13] [14] và để giảm thiểu các phân nhóm đã được thiết lập trước đó [15]. Gần cuối học kỳ đầu tiên, khoảng một tháng trước khi kết thúc môn học, sinh viên hoàn thành đánh giá theo yêu cầu bài toán đưa ra cũng như khảo sát trực tiếp nơi họ làm ( máy móc, thiết bị đo). Các câu hỏi khảo sát bổ sung sẽ được sử dụng để xác định sinh viên nào có thể tạo thành một nhóm như nơi họ sống có gần nhau không, v.v. Kết quả đánh giá và khảo sát, cũng như điểm từ các khóa học sau đó được sử dụng để sắp xếp sinh viên thành các nhóm giống nhau, các nhóm này sẽ được duy trì trong suốt thời gian khóa học.

\section{Các phương pháp tạo động lực nhóm}

\subsection{Phương pháp tiếp cận để thay đổi}

Kể từ khoảng năm 2018, động lực nhóm đã được chính thức trình bày trong các hội thảo của trường, được tổ chức trước các học kỳ mới. Các hội thảo được dẫn dắt bởi phòng kiểm tra chất lượng tại DNTU cùng các giảng viên tham gia giảng dạy. Hội thảo tập trung thảo luận về đặc điểm của các nhóm hoạt động có hiệu quả không, xem xét các lỗi thường gặp của nhóm và phương pháp để giải quyết chúng và cung cấp thêm các phương pháp tối ưu để quản lý xung đột giữa các cá nhân. Những nỗ lực ban đầu để dạy động lực học của nhóm đã đạt được thành công rất hạn chế. Sinh viên ít quan tâm đến tài liệu, đôi khi thiếu tôn trọng người giảng dạy và trong một số trường hợp, họ bình luận trên các diễn đàn, động lực nhóm không phải là một chủ đề quan trọng để các sinh viên học hỏi hoặc thực hành khi ra trường và đi làm tại công ty. Do đó, việc tham gia học rất ít, sinh viên thường bỏ đi trong thời gian nghỉ và không quay trở lại lớp. Với sự thay đổi và cải tiến trong chương trình tích hợp vào năm 2018, cách giảng dạy về tạo động lực nhóm đã được thiết kế lại cho phù hợp. Những thay đổi cơ bản được trình bày trong hội thảo nhằm vào:

- Làm cho các tài liệu giảng dạy phù hợp hơn với từng cá nhân

- Làm cho các nội dung phù hợp hơn với các dự án thiết kế

- Tích hợp nội dung giảng dạy chặt chẽ hơn với phần còn lại của tài liệu khóa học

Các chủ đề đã được thay đổi từ cách trình bày chung sang các cuộc thảo luận cụ thể về sở thích làm việc, mỗi sinh viên sẽ có thể xem cách tiếp cận của riêng họ để nhìn nhận vấn đề, tương tác với những người khác và đưa ra quyết định khác với bạn học của họ. Để làm cho các bài giảng về động lực của nhóm phù hợp hơn với các dự án thiết kế, trong khoảng năm 2019, các bài tập qua dự án khi bắt đầu, các nhóm sẽ được yêu cầu dự đoán khả năng và 
nghĩ ra các phương án để giải quyết chúng. Cuối cùng, trong khi việc giảng dạy tạo động lực nhóm từng bị giảm xuống thì bây giờ đã đạt được thông qua quá trình thay đổi.

\section{Tổng quan về cách tiếp cận}

Việc thực hiện động lực nhóm bao gồm các yếu tố sau:

- Trước khi bắt đầu khóa học, sinh viên hoàn thành bài đánh giá theo bảng trắc nghiệm của giảng viên đưa ra để xác định tính cách của họ. Thông tin này sẽ được người hướng dẫn sử dụng để tạo thành các nhóm cho mỗi dự án.

- Sau dự án đầu tiên, sinh viên hoàn thành một cuộc khảo sát phản ánh và đánh giá hiệu suất của nhóm và bản thân họ trong các khía cạnh khác nhau liên quan đến động lực của nhóm (bảng tổng hợp các câu hỏi khảo sát được cung cấp bên dưới).

- Các bài kiểm tra giữa kỳ và cuối kỳ đánh giá sự hiểu biết của sinh viên và khả năng áp dụng nhóm của họ.

- Sự đoàn kết: Sự đoàn kết tồn tại khi nhóm tạo ra một mục đích chung mạnh mẽ, gắn bó với nhau, và mọi người hỗ trợ lẫn nhau.

\section{Kết quả và thảo luận}

Với sự đổi mới trong chương trình đào tạo theo cách tiếp cận được nêu ở trên làm cho giảng dạy về tạo động lực nhóm phù hợp với mỗi cá nhân sinh viên. Thay vì nói chung chung về cách thức hoạt động của các nhóm, trọng tâm của các hội thảo được tổ chức chuyển sang cụ thể các đặc điểm. Cùng với tài liệu về động lực nhóm được cung cấp đến mỗi sinh viên và với việc vận dụng nhiều phương pháp hơn từ mỗi giảng viên cho nội dung bài học, câu hỏi tại lớp và bài kiểm tra dường như nâng cao hiệu quả của chủ đề bài học hôm đó đến lớp. Bên cạnh đó, những thay đổi khác, chẳng hạn như việc sử dụng rộng rãi phương pháp đánh giá khác như so sánh, hỏi ý kiến và thảo luận phản hồi về quy trình của nhóm đã làm nổi bật mối tương quan tích cực giữa các thành viên trong nhóm và hiệu suất của dự án, tất cả dường như cải thiện sự quan tâm và mức độ tương tác của sinh viên đến tài liệu môn học này.

Hiện sinh viên rất háo hức tham gia vào các hội thảo, sinh viên tự do kết hợp tài liệu về động lực học của nhóm trong lập kế hoạch và báo cáo dự án của họ. Một tập hợp các kết quả từ bảng câu hỏi tùy chọn về động lực học trong khóa học được cung cấp trong Bảng 1 . Đối với mỗi câu hỏi được trình bày trong bảng, sinh viên được yêu cầu trả lời theo Thang điểm Likert từ 1 (hoàn toàn không đồng ý) đến 5 (hoàn toàn đồng ý). Kết quả bảng câu hỏi đưa đến nhận định rằng cách tiếp cận hiện tại được sử dụng để giảng dạy về tạo động lực nhóm đã mang đến sự năng động của nhóm, có hiệu quả trong việc tăng sự chú ý của sinh viên đối với chủ đề, tăng số sinh viên tự tin về bản thân mình với nhóm của họ khi bắt đầu các dự án và tăng cường hiểu biết của sinh viên về cách họ và những người khác làm việc theo nhóm.

Bảng 1. Bảng câu hỏi về động lực nhóm thu thập năm 2019

\begin{tabular}{|l|l}
\hline Câu hỏi & Mức độ hài lòng theo thang điểm Likert \\
\hline
\end{tabular}


Trường Đại học Thủ Dầu Một - Trường Đại học Duy Tân

NXB Tài Chính 2021

\begin{tabular}{|c|c|c|c|c|c|}
\hline & 1 & 2 & 3 & 4 & 5 \\
\hline Các hoạt động tạo động lực nhóm là hữu ích & 0 & 2 & 10 & 41 & 22 \\
\hline Cảm thấy sự năng động sau khi học & 0 & 0 & 22 & 31 & 23 \\
\hline Giảm sự xung đột giữa các cá nhân trong nhóm & 0 & 5 & 17 & 38 & 16 \\
\hline Hội thảo của trường rất hữu ích & 2 & 9 & 23 & 29 & 13 \\
\hline Hiểu thêm về bản thân và cách làm việc hiệu quả & 2 & 2 & 10 & 39 & 27 \\
\hline $\begin{array}{l}\text { Hội thảo về động lực nhóm đã giúp học hỏi } \\
\text { thềm từ các thành viên và cách những người khác làm } \\
\text { việc theo nhóm }\end{array}$ & 0 & 2 & 10 & 37 & 28 \\
\hline Số sinh viên phản hồi ý kiến & 4 & 20 & 92 & 215 & 129 \\
\hline Tỉ lệ phản hồi & $1 \%$ & $4 \%$ & $20 \%$ & $47 \%$ & $28 \%$ \\
\hline
\end{tabular}

\section{Tỉ lệ mức độ hài lòng qua thang đo likert (1-5)}

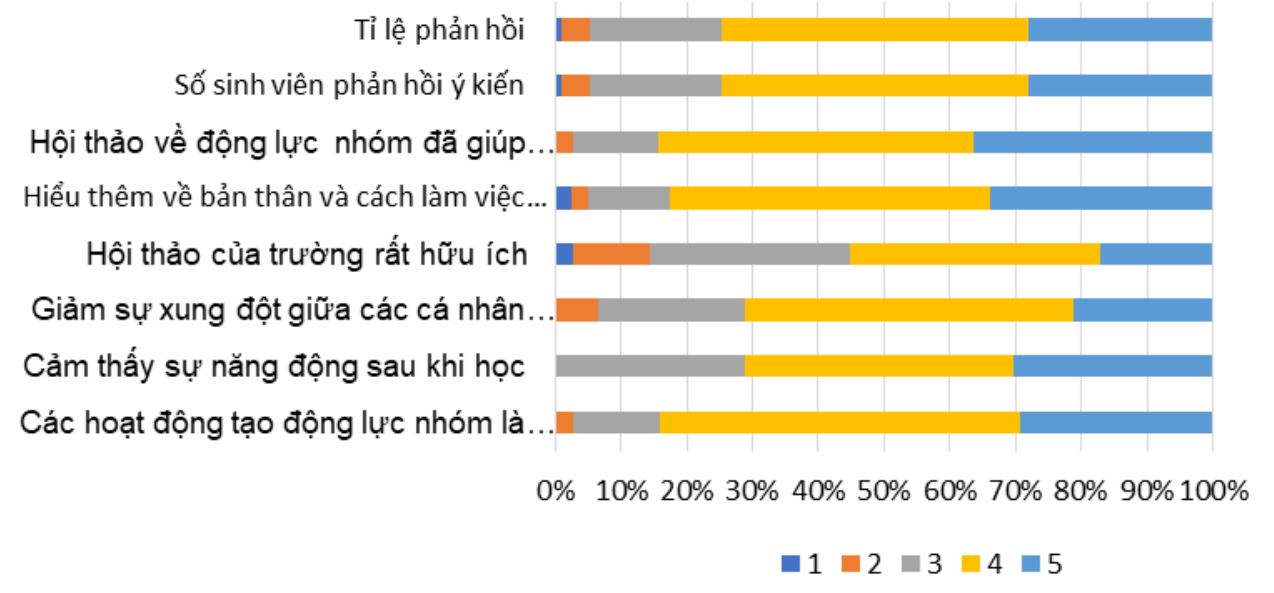

Hinh 1. Biểu đồ minh họa về động lực nhóm thu thập năm 2019

\section{Kết luận}

Trong bài nghiên cứu về giảng dạy tạo động lực nhóm cho sinh viên chuyên ngành cơ khí chế tạo máy chúng ta không thể biết chính xác tác động của mỗi thay đổi được thực hiện trong một nhóm. Có thể, những thay đổi về thái độ của sinh viên là do sự kết hợp của tất cả sự sửa đổi khóa học, cũng như do những thay đổi khách quan trong năm đầu tiên áp dụng việc giảng dạy về tạo động lực nhóm. Dựa trên các phản hồi từ sinh viên, giảng dạy tạo động lực học của nhóm cho sinh viên kỹ thuật là hiệu quả nhất khi:

- Tài liệu được cung cấp theo cách phù hợp với cá nhân mỗi sinh viên.

- Sinh viên và nhóm dự án có thể sử dụng trực tiếp tài liệu từ hệ thống trực tuyến của nhà trường.

- Có các cơ chế phản hồi (chẳng hạn như phản hồi của sinh viên, giảng viên cũng như các cuộc phỏng vấn và thảo luận) để sinh viên nhận thức được và có thể trả lời 
các vấn đề về động lực của nhóm.

- Tài liệu về động lực học của nhóm được cung cấp, trình bày dễ hiểu trong suốt khóa học, bao gồm cả đánh giá và các bảng câu hỏi theo thang điểm Likert.

\section{Tài liệu tham khảo}

[1] Camara, J.E., Carr, B.N., and Grotannn, B.L., "One Approach to Formulating and Evaluating Student Work Groups in Legal Environment of Business Courses", Journal of Legal Studies Education,24(1),1997,pp.1-18.

[2] Chen, Y. and Lou, H., "Students' Perceptions of Peer Evaluation: An Expectancy Perspective", Journal of Education for Business, 79 (5), 2004, pp. 275-282.

[3] Washington Accord, "Rules and Procedures", http://www.washingtonaccord.org [accessed 10 January 2010].

[4] Lovgren, R.H. and M.J. Racer, "Group Dynamics in Projects: Don't Forget the Social Aspects", Journal of Professional Issues in Engineering Education and Practice, October 2000, pp. 156-65.

[5] Bergman, D., Savage, C., Wahstrom, R., and Sandahl, C., "Teaching group dynamics - do we know what we are doing? An approach to evaluation", Medical Teacher, 30, 2008, pp. 55-61.

[6] Crow, M. and Hill, C., "Setting the Stage for Good Group Dynamics in Semester Long Projects in the Sciences", Journal of College Science Teaching, Jan-Feb 2006, pp. 32-5.

[7] Lester, E., Schofield, D., and Chapman, P., "The interaction of engineering 'types': A study of groupdynamics and its relationship to self and peer assessment during computer-based exercises", Engineering Education, Vol. 1, No. 1, 2006.

[8] Fairfield, K.D. and London, M.B., "Tuning into the Music of Groups: A Metaphor for TeamBased Learning in Management Education", Journal of Management Education, Vol. 27, No. 6, December 2003, 654-72.

[9] Babad, E.Y. and Oppenheimer, B.T., "Teaching Group Dynamics in Academic Settings: Basic Dilemmas and Some Tentative Solutions", Teaching of Psychology, Vol. 5, No. 3, Oct 1978, pp. 122-6.

[10] Ostafichuk, P.M., Croft, E.A., Green, S.I., Schajer, G.S., Rogak, S.N., and Hodgson, A.J., "Mech 2: a fully-integrated second-year mechanical engineering curriculum", Proceedings of the 2006 International Mechanical Engineering Education Conference, Beijing, 2006.

[11] Ostafichuk, P.M., Croft, E.A., Green, S.I., Schajer, G.S., and Rogak, S.N. "Analysis of Mech 2: An Award-Winning Second-Year Mechanical Engineering Curriculum", Proceedings of Engineering Education 2008, Loughborough, UK, 2008, pp. 1-12.

[12] Brickell, J.L., Porter, D.B., Reynolds, M.F., and Cosgrove, R.D., "Assigning Students to Groups for Engineering Design Projects: A Comparison of Five Methods", Journal of Engineering Education, July 1994, pp. 259-62.

[13] Wright, D., "Using learning groups in your classroom: a few how-to's", Teaching at UNL (University of Nebraska-Lincoln), 15 (4), 1994.

[14]Feichtner, S. \& Davis, E., "Why some groups fail: a survey of students' experiences with learning groups". The Organizational Behaviour Teaching Review, 9 (4), 1991.

[15] Michaelsen, L.K., Bauman-Knight, A., and Fink, L.D., Team-based learning: A transformative use of small groups in college teaching. Stylus, Sterling, VA., 2004. 